\title{
Calidad de vida relacionada con la salud y consumo de tabaco*
}

\section{Health related quality of life and tobacco consumption}

\section{Armand GRAU \\ Silvia FONT-MAYOLAS ${ }^{2}$ \\ Maria Eugènia GRAS ${ }^{2}$ \\ Rosa SUÑER ${ }^{3}$ \\ Judit NOGUERA ${ }^{4}$}

Fecha de Recepción: 16-06-2006

Fecha de Aceptación: 23-07-2007

\section{RESUMEN}

La calidad de vida relacionada con la salud se ha descrito como un concepto multidimensional que incluye la identificación de síntomas, el estado funcional, la percepción de bienestar psicológico y la percepción general de salud. En el caso de la adicción al consumo de tabaco, la preocupación por la salud actual o futura es uno de los motivos más informados para dejar de fumar. En el presente estudio se analiza la relación entre la percepción de la calidad de vida relacionada con la salud y la etapa de cambio en el abandono del consumo de tabaco propuesta desde el modelo transteórico a fin de mejorar las estrategias de intervención comunitaria y clínica para la cesación tabáquica. Se ha administrado el cuestionario SF-36 a una muestra formada por 201 fumadores y exfumadores. Los datos obtenidos indican peor salud fisica percibida en las etapas de cambio más próximas al abandono de tabaco y una peor salud mental percibida en las etapas con tabaquismo activo. Se comparan los resultados con los observados en otras poblaciones y se discuten las implicaciones a nivel de tratamiento y prevención del tabaquismo.

\footnotetext{
${ }^{1}$ Unidad Coordinadora de Calidad, Formación e Investigación. Hospital de Figueres (Girona).

${ }^{2}$ Instituto de Investigación sobre Calidad de Vida. Universidad de Girona (UdG).

${ }^{3}$ Departamento de Enfermería de la UdG

${ }^{4}$ ABS de Peralada (Girona).

* Esta investigación ha sido realizada con la ayuda económica concedida por la empresa farmacéutica GlaxoSmithKline (GSK) en el año 2005.
} 
Calidad de vida relacionada con la salud y consumo de tabaco

\section{PALABRAS CLAVE}

Calidad de vida relacionada con la salud, Consumo de tabaco, Modelo transteórico, Etapas de cambio.

\section{ABSTRACT}

Health-related quality of life (HRQL) has been described as a multidimensional concept that includes symptoms identification, functional condition, psychological wellbeing perception and general health perception. In the case of nicotine addiction, the concern about current or future health is one of the most reported reasons for smoking cessation. The main goal of this research is to investigate the relationship between HRQL perception and tobacco cessation stage formulated by the Tran theoretical model, with the aim to improve community and clinical interventions. SF-36 questionnaire has been administered to a sample of 201 smokers and ex-smokers. Results show worse perceived physical health in stages of change closer to smoking cessation and worse perceived mental health in stages of change with active tobacco habit. Results are compared with previous studies, while implications for tobacco consumption and prevention are discussed.

\section{KEY WORDS}

Health-related quality of life, Tobacco consumption, Transtheoretical model, Stages of change. 


\section{INTRODUCCIÓN}

Los efectos perjudiciales de fumar en la salud y los elevados costes personales, sociales y económicos asociados al consumo de tabaco, justifican la necesidad de priorizar las intervenciones que faciliten a los fumadores el abandono de este hábito. Precisamente, en más de un $90 \%$ de los fumadores que intentan dejar el hábito, uno de los motivos es la preocupación por su salud actual o futura (Hyland, Li, Bauer, Giovino, Steger y Cummings, 2004).

La calidad de vida relacionada con la salud (CVRS) se ha descrito como un concepto multidimensional que incluye la identificación de sintomas, el estado funcional (físico, psicológico y social), la percepción de bienestar psicológico y la percepción general de salud (Alonso, 2000). Se conoce que la mala salud percibida se encuentra relacionada con un mayor riesgo de mortalidad tanto en hombres como mujeres así como en adultos y ancianos (Kaplan y Camacho, 1983; Mossey y Shapiro, 1982). Asimismo, las personas con mala salud percibida frecuentan más las consultas de atención primaria (Connelly, Philbrick, Smith, Kaiser y Wymer, 1989) y presentan mayor riesgo de hospitalización (Siu, Reuben, Ouslander y Osterwell, 1993), con independencia del estado de salud objetivo. La evaluación de la CVRS se ha llevado a cabo frecuentemente con el cuestionario de salud genérico SF-36 (Sánchez-Sosa y González-Celis, 2006; Ware, Snow, Kosinski y Gandek, 1993). Tillmann y Silcock (1997) han observado que los exfumadores con más de 5 años de abstinencia presentan mejores valoraciones que los fumadores activos en las dimensiones del SF-36 de salud general, vitalidad y salud mental. Woolf, Rotchemich, Johnson y Marsland (1999) detectaron diferencias significativas entre fumadores y no fumadores en todos los dominios del SF-36 de manera que los fumadores muestran peores puntuaciones.

Se ha observado que las personas que han fumado alguna vez puntúan menos en las dimensiones función física, dolor corporal, salud general y vitalidad, del SF-36 (Lyons, Lo y Littlepage, 1994). Es conocido que la CVRS suele irse deteriorando a medida que transcurren los años y es habitual que sea inferior en las personas de más edad. Basándose en el descenso que se observa en estas dimensiones por cada año de edad, se ha establecido que la CVRS de las personas que han fumado alguna vez es equivalente a la que tienen los que nunca han fumado que son 6,6 años más viejos en función física, 15,6 años en dolor, con 14,6 años más en salud general y 14 años más ancianos en vitalidad. Es decir, el tabaco no sólo es responsable de que los fumadores mueran de media 7 años antes que los no fumadores, sino que los años de supervivencia son vividos en peores condiciones subjetivas de salud (Lyons, Lo y Littlepage, 1994; Tarlov, 1994).

Asimismo, durante las primeras seis semanas de cesación tabáquica se ha descrito peor CVRS, atribuida a la abstinencia, en los participantes que recidivan respecto a aquellos que se mantienen abstinentes (Shaw, Coons, Foster, Leischow y Hays, 2001). A los seis meses de conseguir la abstinencia tabáquica se ha observado un aumento en la percepción de salud, de mayor cuantía en la salud física que en la salud mental (Ferrero, Pedrejón, Lozano, Díez, Crespo, Velasco y cols., 2004).

Desde el modelo transteórico de cambio se propone una visión del abandono del consumo de tabaco como un proceso a lo largo de seis etapas: precontemplación (sin intención de abandono en los próximos 6 meses), contemplación 
(intención de abandono en los próximos 6 meses), preparación (intención de abandono en el próximo mes y mínimo un intento de cesación en el último año), acción (abstinencia entre un día y 6 meses), mantenimiento (abstinencia entre más de 6 meses y 3 años) y finalización (abstinencia desde hace más de 3 años) (Cohen, Pederson, Ashley, Bull, Ferrence y Poland, 2002; Prochaska, Norcross y DiClemente, 1994). Estudios realizados para evaluar los pros y contras percibidos de fumar según la etapa han detectado que en las fases más próximas al cambio (contemplación y preparación) los fumadores incrementan su percepción de los contras de fumar (Font-Mayolas, Planes, Gras y Sullman, 2007; Prochaska y cols., 1994). Estudiar las valoraciones de la CVRS en función de la etapa de cambio puede aportar información adicional clave para mejorar los resultados de las estrategias de intervención comunitaria y clínica para el abandono del consumo de tabaco.

El objetivo de la presente investigación consiste en evaluar la percepción de salud que manifiestan los fumadores y exfumadores en las distintas etapas del cambio en el proceso hacia el abandono de la adicción. Es decir, se pretende conocer si cada etapa de cambio cursa con diferentes valoraciones en la percepción de calidad de vida relacionada con la salud a fin de mejorar la eficacia de los abordajes terapéuticos para dejar de fumar (programas comunitarios, intervenciones mínimas e intervenciones psicológicas).

\section{METODOLOGÍA}

\section{Participantes}

La muestra está formada por 201 participantes $(51,7 \%$ hombres y $48,3 \%$ mujeres), con una edad media de 43,9 (dt $=14,5$ ), siendo la edad mínima de 17 años y la máxima de 80 años. Se trata de fumadores activos o exfumadores que han sido visitados en centros de atención primaria o en consultas externas de hospitales de la provincia de Girona.

\section{Instrumentos}

Se ha utilizado un cuestionario de elaboración propia para evaluar la edad, el género y la etapa de cambio tal y como describen Prochaska, Norcross y DiClemente (1994).

La calidad de vida relacionada con la salud se ha valorado mediante el Cuestionario de Salud SF-36 (Alonso, Prieto y Antó, 1995; Ware, Snow, Kosinski y Gandek, 1993; Ware y Gandek, 1998) formado por 36 ítems que cubren las siguientes ocho dimensiones del estado de salud:

Función Física: Indica el grado en que la salud limita las actividades físicas tales como el autocuidado, caminar, subir escaleras, inclinarse, coger o llevar pesos, y los esfuerzos moderados e intensos.

Rol Físico: Expresa el grado en que la salud física interfiere en el trabajo y en otras actividades diarias, lo que incluye el rendimiento menor que el deseado, la limitación en el tipo de actividades realizadas o la dificultad en la realización de actividades.

Dolor Corporal: Refleja la intensidad del dolor y su efecto en el trabajo habitual, tanto fuera de casa como en el hogar.

Salud General: Es la valoración personal de la salud e incluye la salud actual, las perspectivas de salud en el futuro y la resistencia a enfermar. 
Vitalidad: Expresa un sentimiento de energia y vitalidad, frente al sentimiento de cansancio y agotamiento.

Función Social: Evalúa el grado en el que los problemas de salud física o emocional interfieren en la vida social habitual.

Rol Emocional: Valora el grado en el que los problemas emocionales interfieren en el trabajo $u$ otras actividades diarias, lo que incluye la reducción en el tiempo dedicado a esas actividades, el rendimiento menor que el deseado y una disminución del cuidado al trabajar.

Salud Mental: Incluye la depresión, la ansiedad, el control de la conducta y el control emocional, y el afecto positivo en general.

Para cada dimensión, los ítems se codifican, agregan y transforman en una escala desde 0 a 100 (de peor a mejor calidad de vida percibida).

Además de las ocho dimensiones presentadas, el cuestionario SF-36 proporciona información adicional mediante la Evolución Declarada de la Salud: Valora la salud actual comparada con la de un año atrás, a partir de un solo ítem con una escala de 1 a 5 (a menor valor mejor percepción de la evolución del estado de salud actual comparada con la de un año atrás).

\section{Procedimiento}

Se han incluido en el estudio a fumadores activos y exfumadores (menos de diez años desde que dejaron el hábito de fumar) visitados por cualquier problema de salud en centros de atención primaria 0 en consultas externas hospitalarias de la provincia de Girona. Los datos se han recogido desde el 10 de enero hasta el 10 de junio del 2005. La participación ha sido voluntaria previo consentimiento informado. El protocolo fue aprobado por el Comité de Ética en Investigación Clínica del Hospital Josep Trueta de Girona. Las entrevistas se han realizado en el área de consultas externas. Según las caracteristicas del paciente los cuestionarios han sido cumplimentados por un investigador o por el propio paciente, con una comprobación posterior.

\section{RESULTADOS}

\section{Etapa de cambio}

La distribución de la muestra según la etapa de cambio en el abandono del consumo de tabaco es la siguiente: precontemplación ( $\mathrm{n}=62 ; 30,8 \%$ ), contemplación ( $\mathrm{n}=59 ; 29,4 \%)$, preparación $(\mathrm{n}=$ $19 ; 9,5 \%)$, acción ( $\mathrm{n}=21 ; 10,4 \%)$, mantenimiento $(\mathrm{n}=21 ; 10,4 \%)$ y finalización ( $\mathrm{n}=19 ; 9,5 \%$ ). A fin de cumplir las condiciones de aplicación para las pruebas estadísticas, los análisis se realizaron agrupando las etapas de la siguiente forma: fumadores sin intención de cambio (precontemplación), fumadores con intención de cambio (contemplación y preparación) y exfumadores (acción, mantenimiento y finalización).

\section{Salud percibida y etapa de cambio}

\section{Función Física}

La dimensión Función Física obtiene una puntuación media en el global de la muestra estudiada de 82,5 (dt $=20,6)$. En los fumadores que manifiestan intención de cambio se produce un descenso en la percepción de esta dimensión, que comienza a recuperarse cuando dejan de fumar para igualarse e incluso superar las evaluaciones de los precontempladores (tabla 1). Se observa que los fumado- 
res con intención de cambio (contemplación y preparación) tienen peor salud percibida en la dimensión Función Física que los fumadores sin intención de cambio (precontemplación) (t $(56,01)=2,5$; $\mathrm{p}=0,013)$. No se han detectado diferencias estadísticamente significativas en la evaluación de esta dimensión entre los fumadores sin intención de cambio y los exfumadores (acción, mantenimiento y finalización) (t $(14,61)=0,4 ; p=0,70)$. En cambio, al comparar a los fumadores con intención de cambio con los exfumadores se observa que los primeros tienen peor salud percibida en la dimensión Función Física (t $(52,59)=2,9 ; p=0,005)$. camente significativas entre éstos y los fumadores sin intención de cambio $(\mathrm{t}$ $(78,51)=-1,5 ; p=0,12)$ o con intención de cambio (t $(63,09)=0,7 ; p=0,48)$.

\section{Dolor Corporal}

La dimensión de Dolor Corporal presenta una puntuación media de 67,7 (dt $=27,5)$. Los valores de Dolor Corporal muestran un ligero ascenso en los exfumadores (tabla 3 ), pero no se observan diferencias estadisticamente significativas entre los tres grupos (fumadores sin intención de cambio frente a fumadores con intención de cambio (t (194) $=-0,1 ; p$ $=0,89$ ); fumadores sin intención de cam-

Tabla 1: Media y desviación típica de la dimensión Función Física del SF-36 según la etapa del cambio

\begin{tabular}{|l|l|l|l|l|l|l|}
\hline Etapa & Precontemplación & Contemplación & Preparación & Acción & Mantenimicnto & Finalización \\
\hline Media & 86,1 & $n=59$ & $n=19$ & $n=21$ & $n=21$ & $n=19$ \\
$($ di $)$ & $(16,6)$ & 76,2 & 75,8 & 82,9 & 88,3 & 90,8 \\
\hline
\end{tabular}

\section{Rol Físico (RF)}

En la dimensión Rol Físico la puntuación media es de 79,9 (dt $=36,1)$. Los valores de Rol Físico descienden en los fumadores con intención de cambio y en la etapa de Acción, y se mantienen bajos en la etapa de Finalización, a pesar de un incremento considerable durante la etapa de Mantenimiento (tabla 2). Se observa que los fumadores con intención de cambio presentan peor salud percibida en la dimensión Rol Físico que los fumadores sin intención de cambio $(t$ $(53,18)=2,2 ; p=0,030)$. Dada la gran variabilidad existente en las tres etapas del grupo de exfumadores, era previsible que no se hallaran diferencias estadísti- bio frente a exfumadores $(\mathrm{t}(194)=1,5 ; \mathrm{p}$ $=0,11)$ y fumadores con intención de cambio frente a exfumadores $(\mathrm{t}(194)=$ $1,4 ; p=0,16))$.

\section{Salud General}

En la dimensión Salud General la puntuación media es de 60,5 (dt = 19,8). En los fumadores que manifiestan intención de cambio se produce un descenso en la percepción de esta dimensión, que comienza a recuperarse cuando dejan de fumar y alcanza en la etapa de Finalización las puntuaciones más elevadas (tabla 4). No se detectan diferencias estadisticamente significativas en la evalua- 
Tabla 2: Media y desviación típica de la dimensión Rol Físico del SF-36 según la etapa del cambio

\begin{tabular}{|l|l|l|l|l|l|l|}
\hline Etapa & Precontemplación & Contemplación & Preparación & Acción & Mantenimiento & Finalización \\
& $n=62$ & $n=59$ & $n=19$ & $n=21$ & $n=21$ & $n=19$ \\
\hline Media & 87,5 & 76,3 & 69,7 & 70,2 & 96,4 & 68,1 \\
$($ dt $)$ & $(27,8)$ & $(38,9)$ & $(42,1)$ & $(42,2)$ & $(16,3)$ & $(45,2)$ \\
\hline
\end{tabular}

Tabla 3: Media y desviación típica de la dimensión Dolor Corporal del SF-36 según la etapa del cambio

\begin{tabular}{|l|l|l|l|l|l|l|}
\hline Elapa & Precontemplación & Contemplación & Preparación & Acción & Mantenimiento & Finalización \\
& $\mathrm{n}=62$ & $\mathrm{n}=59$ & $\mathrm{n}=1.9$ & $\mathrm{n}=21$ & $\mathrm{n}=21$ & $\mathrm{n}=1.9$ \\
\hline Media & 65 & 66,3 & 65,1 & 74,3 & 67,4 & 77,1 \\
$(\mathrm{dt})$ & $(27,7)$ & $(28,3)$ & $(32,4)$ & $(20,8)$ & $(22,5)$ & $(31,1)$ \\
\hline
\end{tabular}

ción de la salud percibida al comparar fumadores sin y con intención de cambio (t $(45,71)=1,5 ; p=0,13)$ ni al comparar fumadores sin intención de cambio $y$ exfumadores $(\mathrm{t}(104,46)=0,8 ; \mathrm{p}=0,42)$. Los fumadores con intención de cambio evaluaron el componente Salud General significativamente por debajo de los exfumadores (t $(55,09)=2 ; \mathrm{p}=0,042)$.

\section{Vitalidad}

En la dimensión Vitalidad, el global de la muestra presenta una media de 58,9 $(\mathrm{dt}=22,4)$. Los fumadores activos expresan unos niveles de vitalidad muy similares que tienden a incrementarse en los exfumadores (tabla 5). No se observan diferencias estadísticamente significati-

Tabla 4: Media y desviación típica de la dimensión Salud General del SF-36 según la etapa del cambio

\begin{tabular}{|l|l|l|l|l|l|l|}
\hline Etapa & Precontemplación & Contemplación & Preparación & Acción & Mantenimiento & Finalización \\
& $n=62$ & $n=59$ & $n=19$ & $n=21$ & $n=21$ & $n=19$ \\
\hline Media & 61,8 & 57,6 & 53,8 & 58,9 & 62,7 & 72,4 \\
$($ de $)$ & $(17,1)$ & $(17,7)$ & $(26,8)$ & $(18)$ & $(22,3)$ & $(22,2)$ \\
\hline
\end{tabular}


vas en la evaluación de la salud percibida en función de la etapa de cambio (fumadores sin intención de cambio frente a fumadores con intención de cambio (t $(192)=0,3 ; p=0,75)$; fumadores $\sin$ intención de cambio frente a exfumadores $(t)(192)=1,4 ; p=0,37)$; fumadores con intención de cambio frente a exfumadores $(t(192)=1,7 ; p=0,090)$. ción social entre fumadores sin y con intención de cambio (t $(95,98)=1,2$; $\mathrm{p}=0,25)$, ni entre fumadores sin intención de cambio y exfumadores (t $(82,86)=0,9 ; p=0,37)$. En cambio, los exfumadores presentan mayores puntuaciones en esta dimensión que los fumadores con intención de cambio $(\mathrm{t}$ $(72,98)=2 ; \mathrm{p}=0,046)$.

Tabla 5: Media y desviación típica de la dimensión Vitalidad del SF-36 según la etapa del cambio

\begin{tabular}{|l|l|l|l|l|l|l|}
\hline Etapa & Prccontemplación & Contemplación & Preparación & Acción & Mantenimicnto & Finalización \\
& $\mathrm{n}=62$ & $\mathrm{n}=59$ & $\mathrm{n}=19$ & $\mathrm{n}=21$ & $\mathrm{n}=21$ & $\mathrm{n}=19$ \\
\hline Media & 57,7 & 56,1 & 56,8 & 59,7 & 68,5 & 62,3 \\
$(\mathrm{dt})$ & $(21,9)$ & $(25)$ & $(18,2)$ & $(23)$ & $(16,1)$ & $(23,1)$ \\
\hline
\end{tabular}

Tabla 6: Media y desviación típica de la dimensión Función Social del SF-36 según la etapa del cambio

\begin{tabular}{|l|l|l|l|l|l|l|}
\hline Elapa & Precontemplación & Contemplación & Preparación & Acción & Mantenimicnto & Finalización \\
& $\mathrm{n}=62$ & $\mathrm{n}=59$ & $\mathrm{n}=19$ & $\mathrm{n}=21$ & $\mathrm{n}=21$ & $\mathrm{n}=19$ \\
\hline Media & 83,3 & 75,6 & 80,9 & 88,1 & 92,9 & 80,6 \\
$(\mathrm{dt})$ & $(23,3)$ & $(30)$ & $(21,7)$ & $(15)$ & $(16)$ & $(31,8)$ \\
\hline
\end{tabular}

\section{Función Social}

En la dimensión Función Social la puntuación media del total de participantes es de 82,1 (dt = 25,3). Las valoraciones más bajas las encontramos en la etapa de Contemplación (tabla 6) y las más altas en los exfumadores en etapa de Acción y sobre todo de Mantenimiento. No se detectan diferencias significativas en la valoración de la Fun-

\section{Rol Emocional}

En la dimensión Rol Emocional, la puntuación global media es de 82,50 (dt = 33). Los valores más bajos se encuentran en las etapas de cambio más alejadas del abandono del consumo de tabaco. En Contemplación todavía desciende más que en Precontemplación, para posteriormente incrementarse en Preparación y en las etapas incluidas dentro del 
grupo de exfumadores (tabla 7). Se observa que los fumadores sin intención de cambio presentan puntuaciones inferiores en Rol Emocional que los exfumadores (t $(94,63)=2,3 ; p=0,018)$. Los fumadores que se hallan en la etapa de preparación tienen puntuaciones muy similares a las de los exfumadores, mientras que los contempladores se asemejarían más a los precontempladores. Ante esta situación no es de extrañar que no se detecten diferencias estadísticamente significativas entre el grupo de fumadores con intención de cambio y los que no tienen esa intención (t $(90,75)=0,3 ; p=0,75)$ o los exfumadores $(t$ $(66,39)=1,9 ; p=0,058)$.

\section{Salud Mental}

El conjunto de los participantes estudiados obtiene una media de 63,8 (dt =
22,2). Los fumadores activos parten de valores bajos de Salud Mental, que descienden ligeramente en el progreso por las etapas del cambio. Los exfumadores presentan valores más altos en Salud Mental (tabla 8). Se observa que los exfumadores presentan mejores puntuaciones en Salud Mental que los fumadores con intención de cambio ( $\mathrm{t}(192)=2,4$; $\mathrm{p}=0,016)$. En cambio, no se detectan diferencias estadisticamente significativas en la evaluación de esta dimensión entre exfumadores y fumadores sin intención de cambio (t (192)=1,9; $\mathrm{p}=0,058)$ ni entre fumadores sin y con intención de cambio (t $(192)=0,5$; $\mathrm{p}=0,58)$.

\section{Evolución Declarada de la Salud}

La valoración de la salud actual comparada con la de un año atrás se realiza

Tabla 7: Media y desviación típica de la dimensión Rol Emocional del SF-36 según la etapa del cambio

\begin{tabular}{|l|l|l|l|l|l|l|}
\hline Etapa & Precontemplación & Contemplación & Preparación & Acción & Mantenimicnto & Finalización \\
& $n=62$ & $n=59$ & $n=19$ & $n=21$ & $n=21$ & $n=19$ \\
\hline Media & 79,6 & 73,4 & 89,5 & 92,1 & 95,2 & 88,9 \\
$($ dt $)$ & $(32,7)$ & $(40)$ & $(31,5)$ & $(18)$ & $(16)$ & $(32,3)$ \\
\hline
\end{tabular}

Tabla 8: Media y desviación típica de la dimensión Salud Mental del SF-36 según la etapa del cambio

\begin{tabular}{|l|l|l|l|l|l|l|}
\hline Etapa & Precontemplación & Contemplación & Preparación & Acción & Mantenimicnto & Finalización \\
& $\mathrm{n}=62$ & $\mathrm{n}=59$ & $\mathrm{n}=19$ & $\mathrm{n}=21$ & $\mathrm{n}=21$ & $\mathrm{n}=19$ \\
\hline Media & 62,3 & 60,4 & 59,7 & 67,7 & 72,6 & 69,8 \\
$(\mathrm{dt})$ & $(23)$ & $(23,4)$ & $(18,4)$ & $(18,4)$ & $(18,4)$ & $(24,4)$ \\
\hline
\end{tabular}


con una escala de 1 a 5 (a menor puntuación mejor evolución declarada de salud). La puntuación media de los participantes en el presente estudio es de 2,87 ( $\mathrm{dt}=$ $0,86)$. Se aprecia una percepción de peor salud evolutiva cuando hay intención de cambio, sobre todo y en consecuencia en la etapa de Preparación (tabla 9).

Se observa que los fumadores con intención de cambio tienen peor evolución declarada de la salud que los fumadores sin intención de cambio (t (193)=$2,2 ; \mathrm{p}=0,027)$ y que los exfumadores (t $(193)=-4,4 ; p<0,001)$. Asimismo se detecta que los fumadores sin intención de cambio tienen peor evolución declarada de la salud que los exfumadores (t (193) $=-2,3 ; p=0,024)$. trabajo y peor salud actual comparada con la de un año atrás.

Los fumadores con intención de abandonar el consumo de tabaco (etapas de contemplación y preparación) presentan menor evaluación de la CVRS que los exfumadores (etapas de acción, mantenimiento y finalización) en las dimensiones del SF-36: función física, salud general, función social, salud mental y en la evolución declarada de salud. En otras palabras, cuanto más cerca se está de la cesación tabáquica en comparación a quienes se mantienen abstinentes: mayor limitación en las actividades fisicas habituales, menor valoración personal de la salud, mayor interferencia percibida de los problemas de salud en la

Tabla 9: Media y desviación típica de la Evolución Declarada de la Salud del SF-36 según la etapa del cambio

\begin{tabular}{|l|l|l|l|l|l|l|}
\hline Etapa & Precontemplación & Contemplación & Preparación & $\Lambda$ cción & Mantenimiento & Finalización \\
\hline Media & 2,9 & $n=59$ & $n=19$ & $n=21$ & $n=21$ & $n=19$ \\
$(\mathbf{d t})$ & $(0,7)$ & 3,1 & 3,3 & 2,9 & 2,1 & 2,7 \\
\hline
\end{tabular}

\section{CONCLUSIONES}

Los fumadores con intención de abandonar el consumo de tabaco (etapas de contemplación y preparación) presentan inferior valoración de su CVRS que los fumadores sin intención de cambio (etapa de precontemplación) en las dimensiones del SF-36: función física, rol físico y en la evolución declarada de salud. Es decir, una mayor proximidad a la cesación tabáquica en comparación a la lejanía se acompaña de mayor limitación percibida a causa de la salud en las actividades físicas, interferencia en el vida social, mayor puntuación en depresión y ansiedad y peor salud comparada con la de un año atrás.

Los fumadores sin intención de cambio (etapa de precontemplación) presentan inferior valoración de su CVRS en comparación a los exfumadores (etapas de acción, mantenimiento y finalización) en las dimensiones: rol emocional y en evolución declarada de la salud. Es decir, los fumadores lejanos al cambio comparados con los exfumadores informan de mayor interferencia de los problemas emocionales en el trabajo $\mathrm{u}$ otras 
actividades diarias y peor salud actual comparada con la de un año atrás.

Estos resultados están en la línea de anteriores estudios con población suiza y americana en los cuáles se aplicó el cuestionario del balance decisional y se observó que en las etapas que implican intención de cambio (Contemplación y Preparación) se valoran más los efectos perjudiciales sobre la salud del consumo de tabaco o "contras" que en el resto de etapas de cambio (Etter y Pernerger, 1999; Prochaska y cols., 1994). Asimismo los resultados obtenidos en el presente estudio concuerdan con el trabajo de Font-Mayolas y cols. (2007) con población española a la cual se aplicó una entrevista sobre la magnitud y demora percibidas de los contras de fumar para la salud observándose que a mayor proximidad al cambio más importancia se otorgaba a las consecuencias negativas del hábito tabáquico. Posiblemente las dos percepciones, considerar que el tabaco tiene más efectos negativos que placenteros y la peor salud subjetiva, están estrechamente relacionadas y con mutuas influencias.

Del mismo modo los datos obtenidos están en relación con investigaciones previas, en las cuales se ha observado que los fumadores en precontemplación tienden a tener más creencias dirigidas a contrarrestar los conocimientos sobre los perjuicios del tabaco sobre la salud, como pensar que "fumar no debe ser tan malo como dicen si hay tanta gente que ha fumado toda la vida y ha llegado a envejecer sin problemas de salud por el tabaco" (Chapman, Wong y Smith, 1993).

En cuanto a la calidad de vida relacionada con la salud en los exfumadores, los resultados del presente estudio indican en general puntuaciones superiores a las de los fumadores activos desde el primer momento del abandono. En cam- bio, Halpern y Warner (1994) describen que los exfumadores recientes tienen peor salud subjetiva que los fumadores activos, aunque va mejorando en el transcurso de los años siendo superior a la de los fumadores al cabo de un tiempo variable.

Los resultados del presente estudio también concuerdan con los hallazgos de Mulder, Tijhuis, Smit y Kromhout (2001) en una investigación comparativa de fumadores y exfumadores en la cual se observó que los exfumadores percibían mejor calidad de vida relacionada con la salud que los fumadores activos, sobre todo en Salud Mental y Rol Emocional, más que en las dimensiones físicas.

En nuestro conocimiento este estudio es el primero que establece diferencias en la CVRS entre los fumadores activos en función de la etapa del cambio en que se encuentren. Sin embargo, se deben tener en cuenta posibles limitaciones metodológicas de este trabajo. El tamaño reducido de algunos de los grupos no ha permitido llevar a cabo el estudio con las seis etapas de cambio formuladas según el modelo transteórico (Prochaska, Norcross y DiClemente, 1994) y por dicho motivo los análisis estadísticos se han realizado agrupando las etapas de contemplación y preparación (por compartir la intención de cambio) y las etapas de acción, mantenimiento y finalización (por compartir la abstinencia). En futuras investigaciones sería importante incrementar el tamaño de la muestra para poder analizar con mayor profundidad la CVRS en cada una de las etapas de cambio. Además, al tratarse de un estudio transversal, las comparaciones entre etapas se han realizado entre sujetos diferentes. Sería conveniente realizar estudios longitudinales que permitan comparar la evolución de la salud percibida de cada sujeto a lo largo del proceso de abandono 
del consumo de tabaco a fin de controlar la variabilidad individual.

La intervención con programas comunitarios (Agencia de Evaluación de Tecnologías Sanitarias, 2003; Ovejero, 1993, 2000) mediante el incremento de mensajes favorables a la cesación del hábito tabáquico en medios de comunicación y la participación de líderes sociales, políticos y sanitarios, que incidan en la concienciación de la CVRS disminuida en los fumadores y de la ganancia que puede suponer convertirse en exfumador pueden facilitar el paso por las etapas del cambio hasta el abandono.

Por otra parte es probable que los fumadores con intención de cambio, por su peor CVRS, entren más en contacto con los servicios sanitarios. Los profesionales sanitarios en sus intervenciones mínimas (consejo médico, consejo enfermería, consejo psicológico) junto al ofrecimiento de ayuda profesional, deberían aprovechar estas ocasiones para potenciar la percepción de que la salud se está deteriorando a causa del consumo de tabaco e informar de la mejora de la CVRS inmediata al abandono del consumo. De esta forma se podría estar facilitando el tránsito hacia la etapa de acción.
En la misma línea, en las intervenciones psicológicas (individuales o grupales), el incremento de la CVRS que sigue al abandono del consumo de tabaco podría incorporarse como uno de los componentes de los programas de prevención de recaída en las personas que se encuentren en las etapas de acción, mantenimiento y finalización.

En consecuencia, ayudar al fumador a cesar en el consumo de tabaco significaría ayudarlo también a mejorar su salud percibida física y mental, y posiblemente también su salud objetiva, disminuyendo su riesgo de mortalidad y aumentando su calidad de vida.

\section{Miembros del grupo de estudio SPYT (salud percibida y tabaquismo):}

Marian Gimeno, Francesc Tobias, Eva Carpintero, Cristina Montañés, Miguel Cajal, Olga Díaz, Oscar Sánchez, Jerónima Dorado, Ramon Tarrés, Lluís Martínez, Joan Pagès, José Vallejo, Judit Masdevall, Roser Piarnau, Anna Juanola, Esperança Llover, Gemma Fossas, Salvi Sendra, Caty Gelabert, José A. Alvarez, Pepi de las Heras y Pere Comas. 


\section{BIBLIOGRAFÍA}

Agencia de Evaluación de Tecnologias Sanitarias (2003). Evaluación de la eficacia, efectividad y coste-efectividad de los distintos abordajes terapéuticos para dejar de fumar. Madrid: Agencia de Evaluación de Tecnologias Sanitarias. Instituto de Salud Carlos III. Ministerio de Sanidad y Consumo.

Alonso, J. (2000). La medida de la calidad de vida relacionada con la salud en la investigación y la práctica clínica. Gaceta Sanitaria, 14, 163-167.

Alonso, J., Prieto, L. y Antó, J.M. (1995). La versión española del SF-36 Health Survey (Cuestionario de salud SF-36): Un instrumento para la medida de los resultados clínicos. Medicina Clínica (Barcelona) ,104, 771-776.

Chapman, S., Wong, W.L. y Smith, W. (1993). Self-exempting beliefs about smoking and health: differences between smokers and ex-smokers. American Journal of Public Health, 83, 215-219.

Cohen, J.E., Pederson, L., Ashley, M.J., Bull, S.B., Ferrence, R. y Poland, B.D. (2002). Is "stage of change" related to knowledge of health effects and support for tobacco control?. Addictive Behav iors, 27, 49-61.

Connelly, J.E., Philbrick, J.T., Smith, G.R., Kaiser, D.L. y Wymer, A. (1989). Health percepctions of primary care patients and the influence on health care utilization. Medical Care, 27, Supl 3 S99-S109.

Etter, J.F. y Pernerger, T.V. (1999). Associations between the stages of change and the pros and cons of smoking in a longitudinal study of swiss smokers. Addictive Behaviors, 24 (3), 419424

Ferrero, A., Pedrejón, E., Lozano, .J.L., Diez, P., Crespo, J., Velasco, .J y cols., (2004). Mejoría de la función pulmonar y de la calidad de vida en fumadores con abstinencia continua durante 6 meses. Prevención del tabaquismo, 6, supl 1.

Font-Mayolas, S., Planes, M, Gras, M.E. y Sullman, M.J.M. (2007). Motivation for change and the pros and cons of smoking in a Spanish population. Addictive Behaviors, 32, 175-180.

Halpern, M.T. y Warner, K.E. (1994). Differences in former smokers' beliefs and health status following smoking cessation. American Journal of Preventive Medicine, 10, 31-37.
Hyland, A., Li, Q., Bauer, J.E., Giovino, G.A., Steger, C. y Cummings, K.M. (2004). Predictors of cessation in a cohort of current and former smokers followed over 13 years. Nicotine \& Tobacco Research, 6, Supl 3, S363-S369.

Kaplan,G.A. y Camacho, T. (1983). Perceived health and mortality: A nine-year follow-up of the human population laboratory cohort. American Journal of Epidemiology, 117, 292-304.

Lyons, R.A., Lo, S.V. y Littlepage, B.N.C. (1994). Perception of health amongst ever-smokers and never-smokers: a comparison using the SF-36 Health Survey Questionnaire. Tobacco Control, 3, 213-215.

Mossey, J.M. y Shapiro, E. (1982). Self-rated health: a predictor of mortality among the elderly. American Journal of Public Health, 72, 800-808.

Mulder, I., Tijhuis, M., Smit, H.A. y Kromhout, D. (2001). Smoking cessation and quality of life: the effect of amount of smoking and time since quitting. Preventive Medicine, 33, 653-60.

Ovejero, A. (1993). La adicción al tabaco: algunos aspectos psicosociales. Intervención Psicosocial, 2 (5), 95-114

Ovejero, A. (2000). La adicción como búsqueda de identidad: una base teórica psicosocial para una intervención eficaz. Intervención Psicosocial, 9 (2), 199-215.

Prochaska, J.O., Norcross,J.C., y DiClemente, C.C. (1994). Changing for good. New York: Avon Books.

Prochaska, J.O., Velicer, W.F., Rossi, J.S, Goldstein, M.G., Marcus, B.H., Rakowski, W., Fiore, C., Harlow, L.L., Redding, C.A., Rosenbloom, D. y Rossi, S.R. (1994). Stages of change and decisional balance for 12 problem behaviors. Health Psychology, $13,39-46$.

Sánchez-Sosa, J.J. y González-Celis, A.L. (2006). Evaluación de la calidad de vida desde la perspectiva psicológica. En V.E. Caballo (Dir.) Manual para la evaluación clínica de los trastornos psicológicos (pp. 473-492). Madrid: Pirámide.

Shaw, J.W., Coons, S.J., Foster, S.A., Leischow, S.J. y Hays, R.D. (2001). Responsiveness of the 
smoking cessation quality of life (SCQOL) questionnaire. Clinical Therapeutics, 23, 957-969.

Siu, A.L., Reuben, D.B., Ouslander, J.G. y Osterwell, D. (1993). Using multidimensional health measures in older persons to identify risk of hospitalization and skilled nursing placement. Quality of Life Research, 2, 253-261.

Tarlov, A.R. (1994). Tobacco use and the quality of life. Tobacco Control, 3, 196.

Tillmann, M. y Silcock, J., (1997). A comparison of smokers' and ex-smokers' health-related quality of life. Journal of Public Health Medicine, 19, 268273.
Ware, J.E., Snow, K.K., Kosinski, M. y Gandek, B. (1993). SF-36 Health Survey: manual and interpretation guide. Boston, MA: The Health Institute, New England Medical Center, 1993.

Ware, J.E. y Gandek, B. (1998). Overview of the SF-36 Health Survey and the International Quality of Life Assessment (IQOLA) Project. Journal of Clinical Epidemiology, 51, 903-911.

Woolf, S.H., Rotchemich, S.F., Johnson, R.E. y Marsland, D.W. (1999). Is cigarette smoking associated with impaired physical and mental functional status? An office-based survey of primary care patients. American Journal of Preventive Medicine, 17, 134-137. 